\title{
BMJ Open Feasibility of using a transition diabetes team to commence injectable therapies postdischarge from a tertiary hospital: a pilot, randomised controlled trial
}

\author{
Felicity Pyrlis, ${ }^{1}$ Rajna Ogrin, ${ }^{\odot 2,3}$ Sonja Arthur, ${ }^{2}$ Cathy Zhai, ${ }^{2}$ Leonid Churilov, ${ }^{2}$ \\ Sara Baqar, ${ }^{1}$ Jeffrey D Zajac, ${ }^{1,2}$ Elif I Ekinci ${ }^{1,2}$
}

To cite: Pyrlis F, Ogrin R, Arthur S, et al. Feasibility of using a transition diabetes team to commence injectable therapies postdischarge from a tertiary hospital: a pilot, randomised controlled trial. BMJ Open 2019;9:e023583. doi:10.1136/ bmjopen-2018-023583

- Prepublication history for this paper is available online. To view these files, please visit the journal online (http://dx.doi org/10.1136/bmjopen-2018023583).

Received 04 May 2018 Revised 05 August 2019 Accepted 21 August 2019

A) Check for updates

(C) Author(s) (or their employer(s)) 2019. Re-use permitted under CC BY-NC. No commercial re-use. See rights and permissions. Published by BMJ.

${ }^{1}$ Endocrinology, Austin Health, Heidelberg, Victoria, Australia ${ }^{2}$ Medicine, University of Melbourne, Melbourne, Victoria, Australia

${ }^{3}$ Bolton Clarke Research Institute, Melbourne, Victoria, Australia

Correspondence to Dr Felicity Pyrlis; felicity.pyrlis@austin.org.au

\section{ABSTRACT}

Objectives This study aimed to investigate if the use of a transition team was feasible for patients with diabetes being discharged from hospital on injectable diabetes therapies.

Design Pilot, randomised controlled trial.

Setting The trial was conducted between 2014 and 2016 conjointly by a tertiary referral hospital and a community healthcare provider.

Participants Hospital inpatients $(n=105)$ on new injectable diabetes therapies were randomised 1:1 to transition team or standard care. The transition team received in-home diabetes education 24-48 hours postdischarge, with endocrinologist review 2-4 weeks and 16 weeks postdischarge.

Main outcome measures The primary outcome was feasibility, defined by percentage of patients successfully receiving the intervention. Secondary outcomes included safety, defined by hospital readmission and emergency department presentations within 16 weeks postrandomisation, and treatment satisfaction, measured using Diabetes Treatment Satisfaction Questionnaire (DTSQ). Exploratory outcomes included length of stay (LOS) and change in haemoglobin $\mathrm{A} 1 \mathrm{c}(\mathrm{HbA} 1 \mathrm{c})$ throughout the study. Results The intervention was deemed feasible (85\% $(95 \% \mathrm{Cl} 73 \%$ to $94 \%))$. No difference in safety between groups was detected. No difference in change in $\mathrm{HbA} 1 \mathrm{c}$ between groups was detected (standard care median $\mathrm{HbA} 1 \mathrm{C}-1.5 \%$ (IQR $-3.7 \%$ to $-0.2 \%$ ) vs transition team median $\mathrm{HbA} 1 \mathrm{c}-1.9 \%$ (IQR $-3.8 \%$ to $-0.2 \%$ ), $\mathrm{p}=0.83$ ). There was a trend towards reduced LOS in the transition team group (per protocol, standard care median LOS 8 (IQR 5.5-12); transition team median LOS 6 (IQR $3-12), p=0.06)$. There was a significant improvement in patient satisfaction in the transition team (standard care median 10.5 (IQR 8.5-16); transition team DTSQ change version median 15 (IQR 10-17.5), $p=0.047$ ), although interpretability is limited by missing data.

Conclusion This study demonstrated that the use of a novel transition diabetes team is a feasible alternative model of care.

\section{INTRODUCTION}

Despite stability in diabetes-related mortality, ${ }^{1}$ global prevalence of diabetes is rising. ${ }^{2}$
Strengths and limitations of this study

- A strength of this study was the successful collaboration of two large organisations providing support for the transition of care from hospital to home.

- Withdrawal of participants from the transition team in hospital prior to the intervention may reflect reluctance in patients recovering from acute illness to return to hospital or accept health providers entering their home.

- Missing data may have limited interpretability of results.

- Overall, this novel, patient-centred intervention was found to be feasible and acceptable to people living with diabetes.

Consequently, healthcare costs related to diabetes continue to increase over time. ${ }^{3}$

It has been demonstrated that $34 \%$ of hospital inpatients aged over 54 years admitted to a tertiary hospital have diabetes mellitus. ${ }^{4}$ Many studies have demonstrated that inpatients with type 2 diabetes have longer hospital length of stay (LOS) and higher mortality rates compared with those without. ${ }^{5}{ }^{6}$ Factors such as stress hyperglycaemia, medications and inadequate glycaemia management at the time of hospital admission often result in the need for intensification with injectable diabetes therapies. ${ }^{7}$ Furthermore, the hospitalisation period provides an opportunity to identify those who require optimisation of diabetes management. ${ }^{8}$ However, the commencement of injectable therapies can be difficult in the context of concurrent acute illness.

Guidelines recommend that patients are required to demonstrate self-management with injectable therapies prior to hospital discharge. ${ }^{9}$ Diabetes education is crucial in enabling patients to effectively self-manage and assists in optimising glycaemic control 
postdischarge. ${ }^{10}$ However, diabetes education in the hospital setting is subject to a number of limitations, including acute illness, pain and a sense of being overwhelmed. ${ }^{7}$ Home-based diabetes education may prove more effective based on understanding a patient's life context and allowing adaptation of self-management routines, such as timing of injection, sharps disposal and medication storage, to suit the patient's home environment. ${ }^{11}$

Prior to assessing the effectiveness in a full-scale phase III randomised trial, the feasibility and safety of such an intervention needs to be evaluated. ${ }^{12}$ This can be accomplished by conducting a pilot, feasibility study that would assess both feasibility and safety of the intervention in question, the recruitment potential, and would increase clinical experience with the study intervention. ${ }^{12}$

This pilot study, therefore, developed and evaluated the use of a transition team comprising in-home diabetes education by a credentialed diabetes educator (CDE) and early postdischarge assessment by an endocrinologist. We hypothesised that the proposed intervention would be feasible and would not negatively affect patient satisfaction when compared with standard care.

\section{METHODS}

\section{Design}

This pilot, randomised controlled trial was conducted conjointly by a tertiary hospital in metropolitan Melbourne, Australia, and a community-based healthcare provider. Study participants were recruited during inpatient admissions between March 2014 and November 2015 and follow-up continued until March 2016. The trial was stopped after funding was exhausted and sufficient participants were recruited.

\section{Participants}

Hospital inpatients with type 2 diabetes, commencing or altering injectable diabetes therapies, were screened for the study, and randomised to receive the intervention or standard care after providing informed consent. Baseline characteristics of the participants are outlined in table 1. Funding of participants' healthcare is through the Australian government's universal health insurance system, Medicare.

Participant inclusion criteria were the ability to provide informed consent, the presence of type 2 diabetes, age $>18$ years, requirement to commence or change injectable therapies; therefore, requiring a CDE to provide education prior to discharge, medically stable and awaiting diabetes education, reside within a $30 \mathrm{~min}$ travel radius of the hospital, ability to attend hospital for outpatient follow-up and stable glycaemia defined as blood glucose levels between 5 and $15 \mathrm{mmol} / \mathrm{L}$ in the 24 hours prior to randomisation. Patients were excluded if they did not fulfil inclusion criteria.

\section{Randomisation}

Participants were randomised in a 1:1 ratio using permuted block randomisation, by an investigator without patient
Table 1 Comparison of baseline clinical and biochemical characteristics between the control and transition team groups by initial randomisation/intention to treat

\begin{tabular}{lll}
\hline Characteristic & $\begin{array}{l}\text { Standard care } \\
(\mathbf{n = 4 8})\end{array}$ & $\begin{array}{l}\text { Transitions } \\
\text { group }(\mathbf{n}=55)\end{array}$ \\
\hline $\begin{array}{l}\text { Mean age, years (SD) } \\
\text { Number male (\%) }\end{array}$ & $\begin{array}{l}59.4(10.92) \\
\text { Number CALD (\%) }\end{array}$ & $62.96(16.31)$ \\
$\begin{array}{l}\text { Median duration type } 2 \\
\text { diabetes, years (SD) }\end{array}$ & $7.48(7.78)$ & $38(69.1 \%)$ \\
$\begin{array}{l}\text { Mean HbA1c baseline (\%, } \\
\text { SD) }\end{array}$ & $10.81 \%(2.64)$ & $10.06 \%(2.23)$ \\
mmol/mol (mmol/mol, SD) & $83.7(5.36)$ & $86.4(0.87)$ \\
\hline
\end{tabular}

Two-sample Wilcoxon rank-sum (Mann-Whitney) test was used for continuous variables, and Fisher's exact tests was used for binary variables.

CALD, culturally and linguistically diverse; $\mathrm{HbA1c}$, haemoglobin A1c.

contact. Group allocations were concealed by writing allocations on a card, and placing in sealed, unlabelled envelopes, with each consecutive participant given their allocation by a research assistant after informed consent was obtained. Due to the difference in treatment protocols, the study was open-label to the participants and investigators.

\section{Intervention}

Standard care

Participants randomised to standard care were educated by hospital CDEs prior to discharge. Diabetes education regarding injectable therapies in our institution complies with the guidelines of the Australian Diabetes Educators Association. ${ }^{13}$ This comprised education regarding injectable therapy and its storage, and sharps disposal. Additional resources were provided when required. Additional resources included National Diabetes Services Scheme (NDSS) registration, supply of glucometer if required, written patient information regarding diabetes and outpatient follow-up. The NDSS is a scheme administered by the Australian federal government which provides access for people living with diabetes to education and equipment to enhance their ability to effectively self-manage their diabetes. Participants were discharged when medically appropriate and the inpatient team was satisfied that the participant could safely administer injectable therapy. Prior to discharge, appropriate follow-up was organised. General practitioners (GPs) were notified that participants had commenced or changed treatment.

\section{Transition team (intervention)}

The transition team group participants received in-home education to start injectable therapy by the CDE within 24-48 hours of discharge. At the initial visit, the participant was provided with an appropriate glucometer in addition to education regarding medication, storage, 


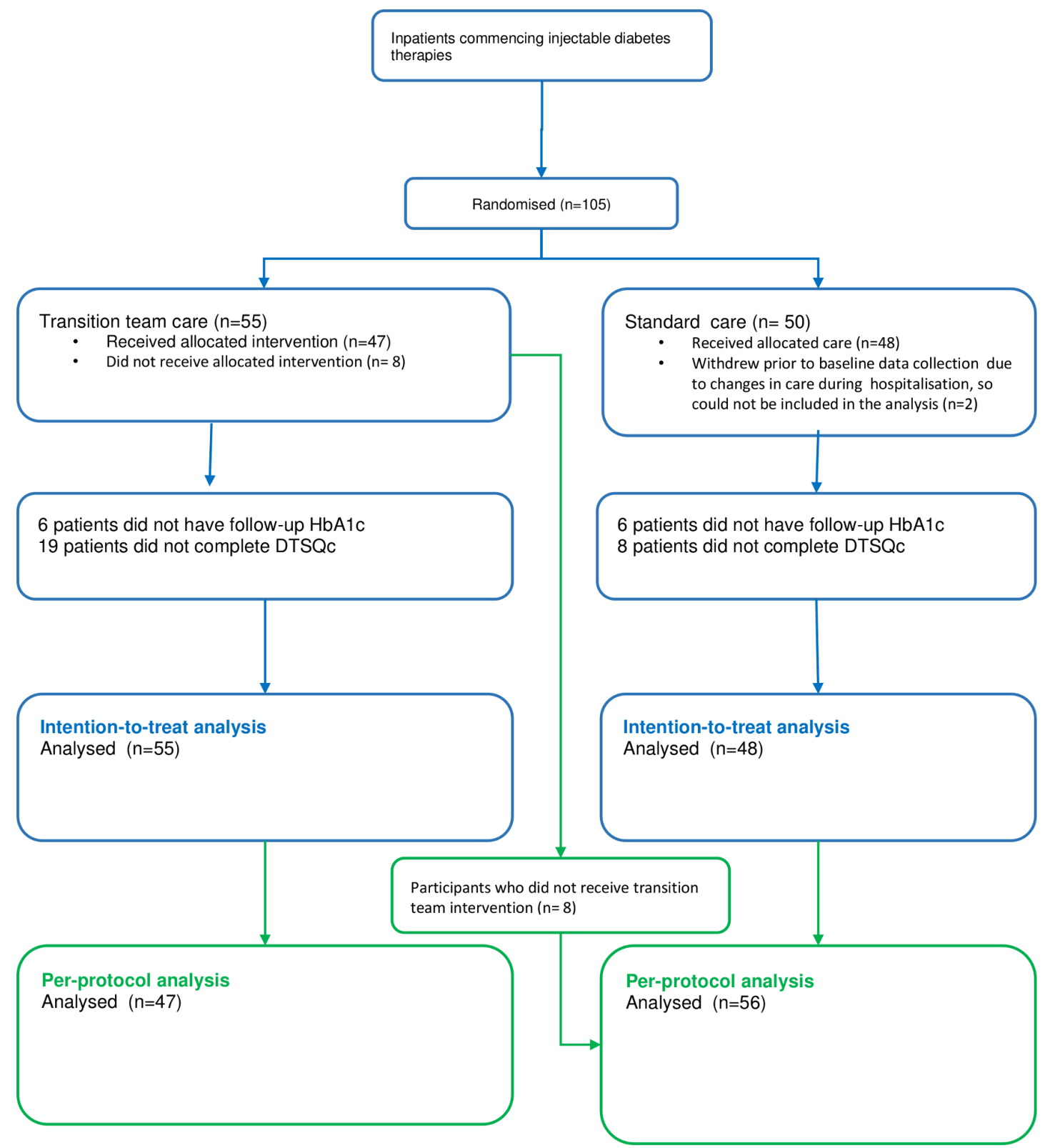

Figure 1 Recruitment and participation flow chart. DTSQc, Diabetes Treatment Satisfaction Questionnaire change version; $\mathrm{HbA1c}$, haemoglobin A1c.

injection technique, sharps disposal, NDSS registration, an education package in the relevant language, and $\mathrm{CDE}$ contact details.

Further contact with participants was based on CDE evaluation of the participant's capacity to self-manage injectable therapy. Once the CDE ascertained that participants were able to self-manage without further intervention, the endocrinologist was notified. Participants were then linked with community CDE services, if necessary, for ongoing monitoring of self-management.

Table 2 Results by intention-to-treat analysis. Differences in hospital readmission rates, change in $\mathrm{HbA} 1 \mathrm{c}$, length of hospital stay and treatment satisfaction according to original randomisation

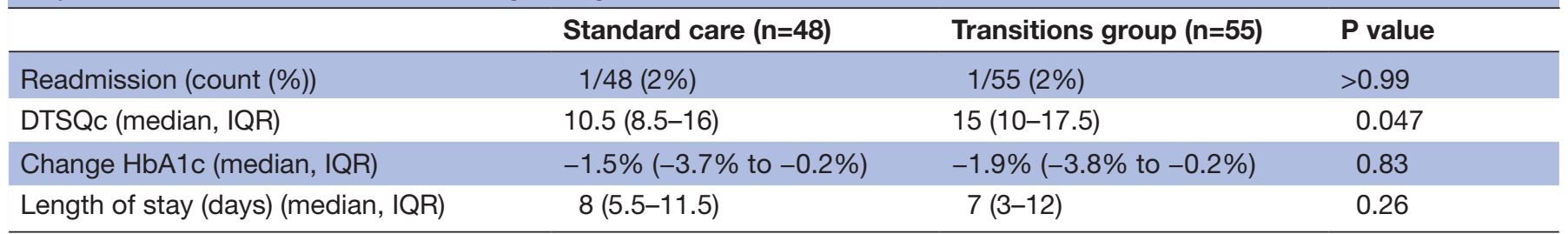

DTSQc, Diabetes Treatment Satisfaction Questionnaire change version; HbA1c, haemoglobin A1c. 
Change in treatment satisfaction (DTSQ)

20

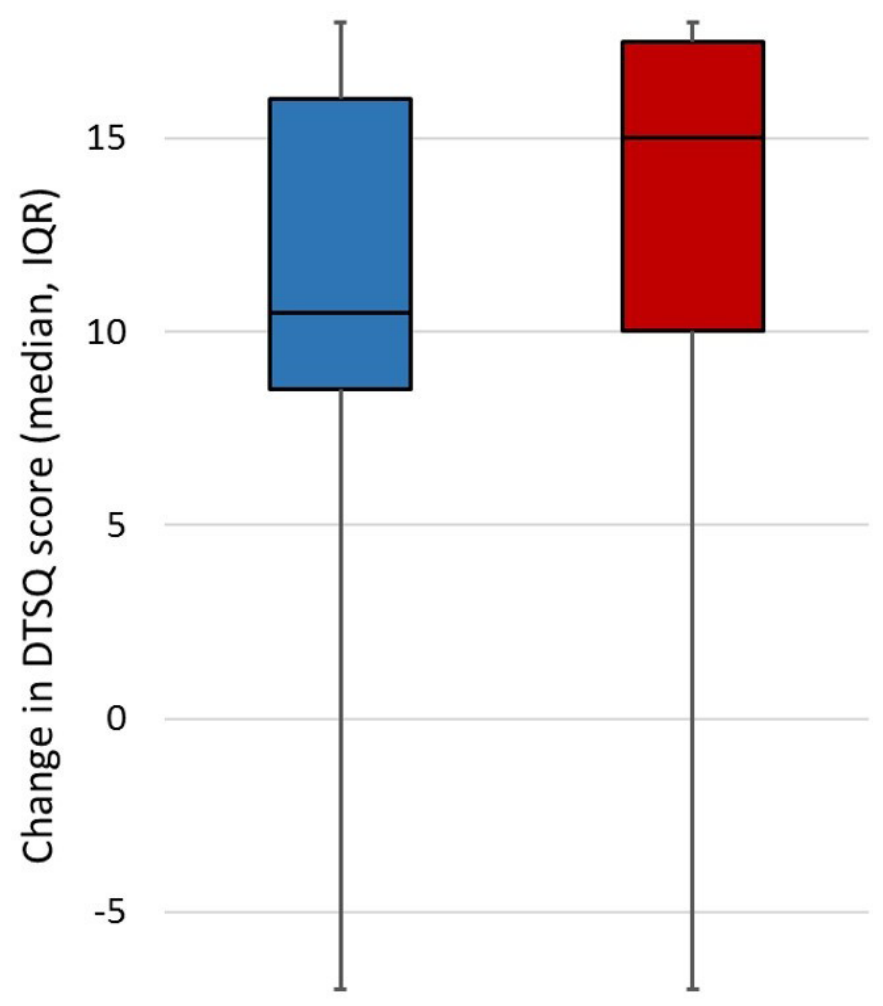

$-10$

\section{$\square$ Control $\square$ Transition Team}

Figure 2 Change in treatment satisfaction (DTSQ). DTSQ, Diabetes Treatment Satisfaction Questionnaire. Median, interquartilerange, minimum and maximum displayed.

Follow-up with the same endocrinologist was provided within 4 weeks and at 16 weeks postrandomisation. Haemoglobin A1c (HbA1c) was assessed at baseline and 16 weeks. The endocrinologist liaised with the participant's GP regarding changes to management and plans for ongoing follow-up after the 16th-week visit.

\section{Data collection}

Baseline demographic, medication and medical data were collected and participants completed the Diabetes Treatment Satisfaction Questionnaire status version (DTSQs) at enrolment. Whether the patient was from a culturally and linguistically diverse background was recorded. Follow-up data were collected at 16 weeks postrandomisation, including rates of hospital readmissions and emergency presentations, length of hospital stay (LOS), glycaemic management as measured by HbAlc and treatment satisfaction using the DTSQ change version (DTSQc). Initial attempts to collect DTSQc questionnaires in the standard care group was by reply paid mail; however, after a limited response rate using this method,
A
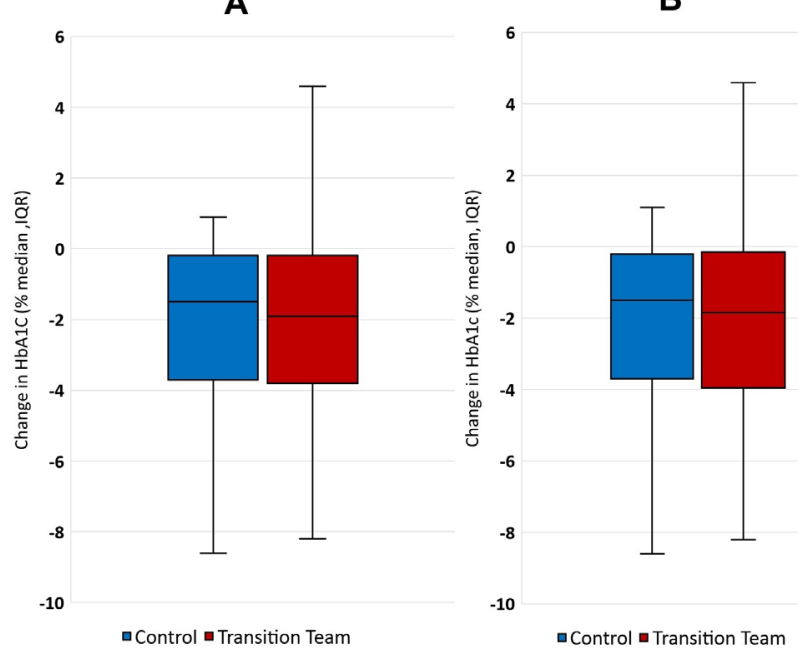

Figure 3 (A) Change in $\mathrm{HbA} 1 \mathrm{c}(\%)$ intention-to-treat analysis. (B) Change in $\mathrm{HbA1c}$ (\%) per-protocol analysis. $\mathrm{HbA1c}$; haemoglobin A1c. Median, interquartilerange, minimum and maximum displayed.

patients were contacted by phone. Study participation ceased at the 16th-week endocrinology appointment, and final data collection occurred. Further follow-up for ongoing diabetes management was arranged at the conclusion of the study.

\section{Ethics}

Each participant provided written informed consent.

\section{Outcomes}

The primary outcome measured was feasibility (proportion of participants in the transition team group completing the intervention as per protocol). Secondary outcomes were safety, as defined by hospital readmission and emergency department presentations within 16 weeks postrandomisation, and patient satisfaction with care (measured by DTSQ). Exploratory outcomes were change in HbAlc and length of hospital stay (days).

\section{Sample size determination}

Due to the pilot nature of the study, the sample size estimation was based on precision arguments: assuming the feasibility of transition team intervention being 0.9 (ie, that $90 \%$ of participants randomised into transition team group would be able to complete the intervention as per protocol), ${ }^{14}$ the sample of 55 participants randomised to the transition team group provides the precision (desired half-width of the $95 \%$ CI) of 0.08 .

The same number of participants was to be randomised to the standard care group, thus ensuring $80 \%$ power to detect potential medium-to-large effects of transition team intervention compared with the standard care (Cohen's $d=0.55$ ), assuming the settings of alpha $=0.05$. Thus, the total sample size for this study was proposed as 110 participants. 
A

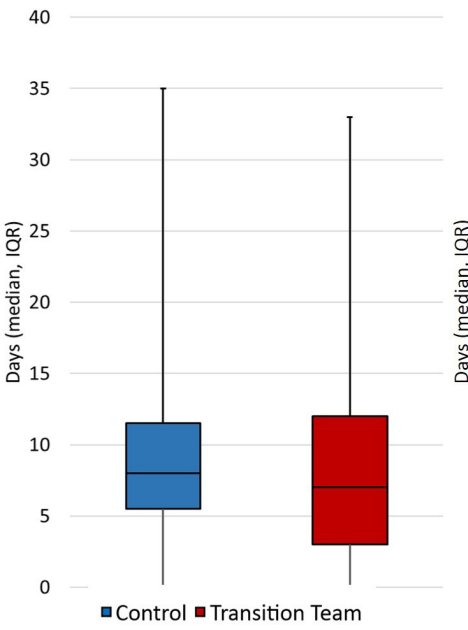

B

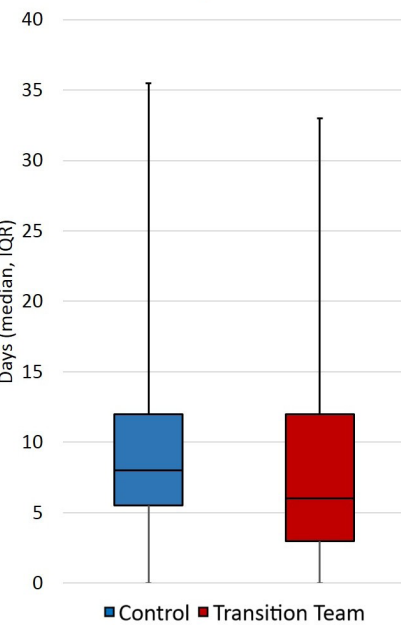

Figure 4 (A) Length of hospital stay (days) by intentionto-treat analysis. (B) Length of hospital stay (days) by perprotocol analysis. Median, interquartilerange, minimum and maximum displayed.

\section{Statistical methods}

The demographic and clinical characteristics of participants were summarised as medians (IQRs) for continuous variables and counts (proportions) for categorical variables.

The feasibility of the intervention was estimated as a proportion of participants in the transition team group completing the intervention as per protocol with corresponding $95 \%$ CI.

The difference in safety profiles (diabetes-related hospital presentation or admission) between two groups was investigated using Fisher's exact test.

DTSQ outcomes were analysed using Wilcoxon-MannWhitney test and a median regression model with the DTSQ score at 16 weeks postrandomisation as an output and treatment group and baseline DTSQ score as inputs. Sensitivity analysis was conducted by including the auxiliary variables, demonstrating a significant association with the DTSQ data being missing, into the median regression model.

Differences in change in HbAlc and LOS between groups were investigated using Wilcoxon rank-sum test.

Statistical analysis was performed using STATA software v14IC (StataCorp, College Station, TX, USA).

All statistical tests were two-sided and were performed at a significance level of $\alpha=0.05$.

Statistical analysis was performed on both intentionto-treat and per-protocol bases. Per-protocol analysis was deemed necessary to account for the patients who were initially assigned to transition team but withdrew prior to intervention; in this situation, they received standard care.

\section{Patient and public involvement}

Patients must be actively involved in changes to diabetes management, and require education regarding therapy changes and administration of injectable medications. Effective education of patients can be difficult in the hospital setting, particularly in the setting of concurrent acute illness. It is subject to a number of limitations, including acute illness, pain and a sense of being overwhelmed in hospital. These clinical observations contributed to the formulation of our research questions; however, there was no direct patient involvement in this.

Patients were not involved in the original study design, and involvement of patients in recruitment was impractical as patients were hospitalised at the time of recruitment. A qualitative analysis of a subgroup of patients' experiences and their perceptions of the intervention was performed, and this will be reported separately.

\section{RESULTS}

A total of 105 participants were randomised to transition team or standard care. Following initial dropouts, 103 participants received the intervention or standard care (figure 1). A total of 55 participants randomised to the transition team and 48 participants randomised to receive standard care were included in the per-protocol analysis (figure 1). Participants randomised to the transition team withdrew for a variety of reasons, such as decisions to change treatment, change of discharge destination and changes to clinical status. Of these, five participants withdrew following the home visit by the CDE but prior to completing the endocrinologist component of the intervention. For the purposes of the per-protocol analysis, these participants crossed over to the standard care group.

One participant in the transition team group was unable to be followed-up as they were being actively palliated for

Table 3 Results by per-protocol analysis. Differences in hospital readmission rates, change in HbA1c, length of hospital stay and treatment satisfaction according to management received

\begin{tabular}{llcc}
\hline & Control group (n=56) & Transitions group (n=47) & P value \\
\hline Readmission (count (\%)) & $1 / 56(2 \%)$ & $1 / 47(2 \%)$ & $>0.99$ \\
DTSQc (median, IQR) & $10.5(8.5-16)$ & $15(10-17.5)$ & 0.047 \\
Change HbA1c (median, IQR) & $-1.5 \%(-3.7 \%$ to $-0.2 \%)$ & $-1.85 \%(-4 \%$ to $-0.2 \%)$ & 0.85 \\
Length of stay (days) (median, IQR) & $8(5.5-12)$ & $6(3-12)$ & 0.06 \\
\hline
\end{tabular}

DTSQc, Diabetes Treatment Satisfaction Questionnaire change version; HbA1c, haemoglobin A1c. 
terminal malignancy, and one died before the completion of the trial, for reasons unrelated to diabetes. One participant withdrew from the standard care group and one died before final data analysis in this group.

Baseline characteristics of participants are outlined in table 1. No differences between groups at baseline were identified.

\section{Feasibility}

A total of 47 out of 55 participants in the transition team group completed the study as per protocol $(85 \%, 95 \% \mathrm{CI}$ $73 \%$ to $94 \%)$.

\section{Safety}

There was one hospital presentation in each group (table 2), one for hypoglycaemia (standard care) and one for inability to cope with insulin management at home due to change in social circumstances (transition team). Neither group participant was admitted.

\section{Patient satisfaction}

A significant improvement in satisfaction with diabetes treatment was demonstrated (DTSQc transition team median 15 (IQR 10.0-17.5), standard care median 10.5 (IQR 8.5-16.0) Wilcoxon-Mann-Whitney, p=0.047) (figure 2 and table 2). On the analysis adjusted for the baseline DTSQs value, the transition team median DTSQc value was 4 points higher than the standard care median (95\% CI 0.25 to $7.75, \mathrm{p}=0.037$ ). The follow-up DTSQc was completed by $40(16.7 \%$ missing $)$ of the standard care patients and 36 ( $34.5 \%$ missing) of the transition team patients; thus, the 'missingness' of the data was not likely to be random. On the sensitivity analysis adjusted for the variables significantly associated with the missing DTSQc data at 16 weeks, the results remained qualitatively similar.

\section{Haemoglobin A1c}

No statistically significant difference in change in HbAlc (standard care median HbAlc $-1.5 \%$ (IQR $-3.7 \%$ to $-0.2 \%)$ vs transition team group median HbA1c $-1.9 \%$ (IQR $-3.8 \%$ to $-0.2 \%$ ), $\mathrm{p}=0.83$ ) was observed (figure 3 and table 2). An equal number of patients ${ }^{8}$ in each group did not have an HbA1c measurement at 16 weeks.

\section{Length of stay}

There was a trend towards a reduced LOS in the transition team group when analysed on a per-protocol basis (standard care median LOS 8 (IQR 5.5-12.0), transition team median LOS 6 (IQR 3.0-12.0), p=0.06) (figure 4 and table 3$)$.

\section{DISCUSSION}

\section{Key findings}

The most important finding in this trial was that a transition team to initiate injectable diabetes therapies following discharge is a feasible model of care. These data suggest that a transition team is safe and acceptable with a trend towards reduced length of hospital stay. Moreover, patients randomised to the transition team group had greater treatment satisfaction as demonstrated by a greater difference in DTSQc score.

\section{Relationship with previous studies}

The results pertaining to the quality of this intervention are supported by results from other studies examining home-based care in diabetes. The quality markers of this intervention include feasibility and objective measures of medical indices, including readmission and emergency presentation rates, change in HbAlc from baseline and LOS, and patient-reported outcomes.

We demonstrated a trend towards $(\mathrm{p}=0.06)$ reduced LOS in the transition team when analysed on a per-protocol basis. Future studies with greater numbers may demonstrate statistically significant reductions in length of hospital stay.

We demonstrated significant improvements in treatment satisfaction in the transition team group. The DTSQ is widely used in clinical trials and validated in several languages. The DTSQs evaluates baseline satisfaction with diabetes treatment and the DTSQc evaluates the impact of an intervention on satisfaction with treatment. ${ }^{15-18}$ Interpretability of this parameter is limited by missing data, and there is potential for bias due to some data being obtained over the phone by a research assistant, rather than in person.

Analysis of HbAlc at baseline and 16 weeks revealed a significant treatment effect with HbAlc reduction approaching $2 \%$ in both groups. Importantly, there was no statistical difference detected between the reduction in HbA1c in the transition team and standard care groups.

Other quality outcomes assessed included emergency department presentations and hospital readmissions. There were no significant differences in our study. This suggests that patient safety is unlikely to be compromised by delivery of home-based education. However, given the limited literature in this field, further studies with greater numbers would be necessary to validate these findings.

\section{Study implications}

Hospitalisation in patients with type 2 diabetes provides an opportunity to intervene to improve outcomes over the course of the disease. Escalating rates of diabetes necessitate the development of feasible alternative models of care, with the potential to improve clinical outcomes and healthcare costs. This study has investigated one such option, and has demonstrated feasibility, improved treatment satisfaction and a trend to reduced LOS, with no safety concerns detected.

\section{CONCLUSION}

The results of this novel pilot study suggest that the use of a transition team provides a feasible alternative model of care for patients with type 2 diabetes requiring initiation of injectable therapies. More research is necessary 
to validate these findings in larger populations, and to ascertain whether it may lead to reduced LOS and healthcare costs.

Acknowledgements The Diabetes Treatment Satisfaction Questionnaire (DTSQ) used in this publication is owned by Professor Clare Bradley and sourced from HPR Ltd. The team gratefully acknowledges Paul Steel, CDE, for providing the in-home diabetes education to the intervention group.

Contributors FP: involved in the development of the trial and clinical management of participants, and performed final write-up. RO: involved in the development of protocol and supervision of the trial, and reviewed final write-up. SA: logistics of the trial (research assistant), data collection and statistical analysis. CZ: logistics of the trial (research assistant), final data collection and statistical analysis. SB: recruitment of participants and management of logistics of the trial (research assistant), and data collection and analysis. LC: statistical analysis. JDZ: supervisory/advisory role. EE: development of protocol, supervisory and advisory role during the trial, and assisted with statistical analysis and final write-up.

Funding The Lord Mayor's Charitable Foundation and the Estate of the Late Glen W A Griffiths funded this project. Elif I Ekinci was supported by the Australian National Health and Medical Research Council (NHMRC) Early Career Fellowship, Viertel Clinical Investigatorship, Royal Australasian College of Physicians (RACP) Fellowship and Sir Edward Weary Dunlop Medical Research Foundation research grant.

Competing interests None declared.

Patient consent for publication Obtained.

Ethics approval Ethics approval was obtained from the Austin Health human research ethics committee (reference LNR/13/Austin 179) and the community healthcare provider ethics committee.

Provenance and peer review Not commissioned; externally peer reviewed.

Data availability statement Data are available on reasonable request.

Open access This is an open access article distributed in accordance with the Creative Commons Attribution Non Commercial (CC BY-NC 4.0) license, which permits others to distribute, remix, adapt, build upon this work non-commercially, and license their derivative works on different terms, provided the original work is properly cited, appropriate credit is given, any changes made indicated, and the use is non-commercial. See: http://creativecommons.org/licenses/by-nc/4.0/.

\section{REFERENCES}

1. Australian Institute of Health and welfare. Diabetes deaths. Canberra: AlHW, 2010.

2. Guariguata L, Whiting DR, Hambleton I, et al. Global estimates of diabetes prevalence for 2013 and projections for 2035. Diabetes Res Clin Pract 2014;103:137-49.
3. Australian Institute of Health and welfare. Type 2 Diabetes in Australia's children and young people: a working paper. Canberra: AlHW, 2014 (AlHW Cat. No. CVD 53; Diabetes Series No 21).

4. Nanayakkara $\mathrm{N}$, Nguyen $\mathrm{H}$, Churilov $\mathrm{L}$, et al. Inpatient $\mathrm{HbA} 1 \mathrm{c}$ testing: a prospective observational study. BMJ Open Diabetes Res Care 2015;3:e000113.

5. Medhi M, Marshall MC, Burke HB, et al. Hba1C predicts length of stay in patients admitted for coronary artery bypass surgery. Heart Dis 2001;3:77-9.

6. Baker ST, Chiang CY, Zajac JD, et al. Outcomes for general medical inpatients with diabetes mellitus and new hyperglycaemia. Med $J$ Aust 2008;188:340-3.

7. Korytkowski MT, Koerbel GL, Kotagal L, et al. Pilot trial of diabetes self-management education in the hospital setting. Prim Care Diabetes 2014;8:187-94.

8. Schäfer I, Pawels M, Küver C, et al. Strategies for improving participation in diabetes education. A qualitative study. PLoS One 2014;9:e95035.

9. Joint British Diabetes Societies for Inpatient Care. Discharge planning for adult inpatients with diabetes, 2015.

10. Wexler DJ, Beauharnais CC, Regan S, et al. Impact of inpatient diabetes management, education, and improved discharge transition on glycemic control 12 months after discharge. Diabetes Res Clin Pract 2012;98:249-56.

11. de Carvalho Torres H, dos Santos LM, de Souza Cordeiro PMC. Home visit: an educational health strategy for self-care in diabetes. Visita domiciliária: estratégia educativa em saúde para $\mathrm{O}$ autocuidado em diabetes. Acta Paulista de Enfermagem 2014;27.

12. Thabane L, Ma J, Chu R, et al. A tutorial on pilot studies: the what, why and how. BMC Med Res Methodol 2010;10.

13. Australian Diabetes Educators Association (ADEA). Clinical guiding principles forSubcutaneous injection technique, 2015. Available: https://www.adea.com.au/wp-content/uploads/2015/11/InjectionTechnique-Final-digital-version2.pdf

14. Pérez A, Reales P, Barahona MJ, et al. Efficacy and feasibility of basal-bolus insulin regimens and a discharge-strategy in hospitalised patients with type 2 diabetes--the HOSMIDIA study. Int $\mathrm{J}$ Clin Pract 2014;68:1264-71.

15. Bradley C, Lewis KS. Measures of psychological well-being and treatment satisfaction developed from the responses of people with tablet-treated diabetes. Diabet Med 1990;7:445-51.

16. Bradley C, Speight J. Patient perceptions of diabetes and diabetes therapy: assessing quality of life. Diabetes Metab Res Rev 2002;18:S64-S69.

17. Bradley C. Diabetes treatment satisfaction questionnaire. change version for use alongside status version provides appropriate solution where ceiling effects occur. Diabetes Care 1999;22:530-2.

18. Bradley C, Plowright R, Stewart J, et al. The diabetes treatment satisfaction questionnaire change version (DTSQc) evaluated in insulin glargine trials shows greater responsiveness to improvements than the original DTSQ. Health Qual Life Outcomes 2007;5:57. 